\title{
Breast 3D: An Augmented Reality System for Breast CT and MRI
}

\author{
Benjamin Allison, Xujiong Ye, Faraz Janan \\ School of Computer Science \\ University of Lincoln \\ Lincoln, Country \\ ballison@lincoln.ac.uk
}

\begin{abstract}
Adoption of Virtual Reality (VR), Augmented Reality (AR) and Mixed Reality (MR) - known collectively as Extended Reality (XR) devices has been rapidly increasing over recent years. However, the focus of XR research has shown a lack of diversity in solutions to the problems within medicine, with it being predominantly focused in augmenting surgical procedures. Whilst important, XR applied to aiding medical diagnosis and surgical planning is relatively unexplored. In this paper we present a fully functional mammographic image analysis system, Breast3D, that can reconstruct MRI and CT scan data in XR. With breast cancer Breast Imaging-Reporting and Data System (BI-RADS) risk laxicon, early detection and clinical workflow such as Multi-disciplinary team (MDT) meetings for cancer in mind, our new mammography visualization system reconstructs CT and MRI volumes in a real 3D space. Breast3D is built upon the past literature, and inspired from research for diagnosis and surgical planning. In addition to visualising the models in MR using the Microsoft HoloLens, Breast3D is versatile and portable to different XR head-mounted displays such as HTC Vive. Breast3D demonstrates the early potential for XR within diagnostics of 3D mammographic modalities, an application that has been proposed but until now has not been implemented.
\end{abstract}

Index Terms-Extended Reality, Mixed Reality, Augmented Reality, Breast Cancer, Computer Aided Diagnosis

\section{INTRODUCTION}

Diagnostic radiology constitutes the largest pool of medical images in clinical practice. A large community of image processing experts, medical physicists, and computer vision experts develop methods and algorithms aiming to help clinicians at the point of care. Magnetic resonance imaging (MRI) is used to get an in-depth analysis of potential abnormalities in breasts. It evidently overcomes the limitations of a conventional mammogram, particularly in finding tumours in dense breasts. MRI builds 3D reconstructed stack of images that can be zoomed in and out while projected on a 2D 'flat' screen. However, the intrinsic value of 3D data in a 2D representation poses a fundamental challenge and investigation gap in exploiting its full clinical and research potential.

Breast cancer screening is routine clinical practice in the UK (and several other countries around the world) for postmenopause and high-risk women. It has proved vital in reducing the mortality rate via early detection and diagnosis of breast cancer, and in prognosis for increasing the life expectancy. It is estimated that without screening in early detection, there would be 360.1 years of life lost per 1,000 women [1]. With early diagnosis patients have a improved prognosis, reducing the overall cost of the treatment, and widening the available treatments. A system to aid the screening process in the diagnosis of breast cancer has been proposed for XR [2], however, its potential applicability within clinical settings are yet to be investigated.

Early validation of extended reality (XR) devices have helped grow its research within the medical sector. But there is great potential still in developing the fundamental techniques in XR to aid radiologists in diagnosis. With XR's ability to interact and anchor 3D objects within the real-world these technologies are suited to these problems. Whilst other approaches to 3D visualisation have been presented, few of them like XR address the challenges faced by radiologist in providing a practical and ergonomic solution.

\section{EXTENDED REALity in BREASt IMAGE ANALYSiS}

$\mathrm{XR}$ is in the early years of its applications to breast image analysis. It's potential has started to be identified for radiology in the past few years [3], and some have began to investigate the applicability [4] with utilising this technology in radiology and oncology. Most of the work in the area in general is within surgical planning, nevertheless, specifically for breast cancer using MRI data. However, there are even fewer examples of $\mathrm{XR}$ in breast cancer radiology [5].

As previously mentioned there are few proof-of-concept AR systems within mammography. XR technology has yet to reach its full potential for radiology, preliminary systems have started to be proposed for breast cancer diagnosis. The system D3D [5]-[7] is the only known XR system for breast cancer diagnosis. D3D showed promising results demonstrating the depth perception and focal point convergence of XR systems in medical imaging. They noted in their findings that they are yet to determine the utility of the system with the clinical practice. In addition, they are yet to demonstrate the improvement upon the visualisation of $\mathrm{XR}$ over $2 \mathrm{D}$ representations. The system achieves basic volumetric rendering, with an interaction system of movements using a joystick. Whilst functional, we believe this is challenging to implement in a clinical setting in its current form, as compared to 'controlerless' environments [4], [8].

Our paper proposes an XR system, Breast 3D, for use in mammography. It improves upon the current practice as well 
as extend to other radiological and oncological sub specialities. We show with our system we are able to produce an apparatus for visualising and interacting with radiological data using Microsoft Hololens. The application we propose has much further potential for distributed systems. We present a few application where Breast 3D could be useful for breast cancer image analysis in future.

\section{A. XR Radiomics}

The potential impact of XR on radiology is discussed in [3], [4] and [5]. However, there is greater potential in the applicability of XR in breast and medical image analysis that has yet to by exploited. Breast screening as proven by [1] helps in the early detection of breast cancer. A significant practice within breast cancer screening is assessment of breast cancer risk. It is found that within the years 2018-2019 in the UK, 8.8 cases of breast cancer were found per 1000 women screened in this process. XR visualisation of data will open doors for extracting new radiomic features using data-characterisation algorithms, that are otherwise hard to see using a naked eye.

\section{B. Breast Cancer Risk Assessment}

XR systems offer potential to redefine approaches to quantify breast density classification, commonly the breast imaging-reporting and data system (BI-RADS) [9]. BI-RADS categorises patients into four risk categories A, B, C and D from breast predominantly fatty to extremely dense. BIRADS correlate with the risk of a patient developing breast cancer, for example [10] in women aged 60-74, a patient has relative risk of 1.42 in BI-RADS, meaning they are $42 \%$ more likely to develop breast cancer when compared to the average risk of that age group. When comparing women aged $50-59$ [10] finds that women are 2.1 times higher at risk of developing breast cancer having been assessed as BI-RADS D as compared to BI-RADS A. The leading software for breast density assessment are Volpara [11] and Quantra [12]. To our knowledge there is no system that uses volumetric data from $\mathrm{XR}$ to quantify breast density into fat and non-fat.

\section{Cancer Masking}

Higher BI-RADS score pose a high risk of developing breast cancer. However, commonly seen within patients of BI-RADS $\mathrm{C}$ and $\mathrm{D}$, a developing mass could be masked. Breast tissue can be comprised of complex patterns of focal densities, resulted from multi-layered and multi-oriented tissue composition [13]. This has a sever impact in detection masses hiding behind a dense parenchyma, particularly in dense breasts [14] where human perception fail to penetrate through focal densities. It is estimated that $20 \%$ of invasive breast cancers are missed [15], which are later detected as interval cancers. XR can provide a better approach to visualisation and volume exploration of 3D mammographic CT and MRI volumes. With an XR system, masses can be unmasked as the user can view from all angles. In cases where dense tissue obscures the mask a different viewing angle may be used to confirm developing asymmetries. In addition, by giving the user a better method

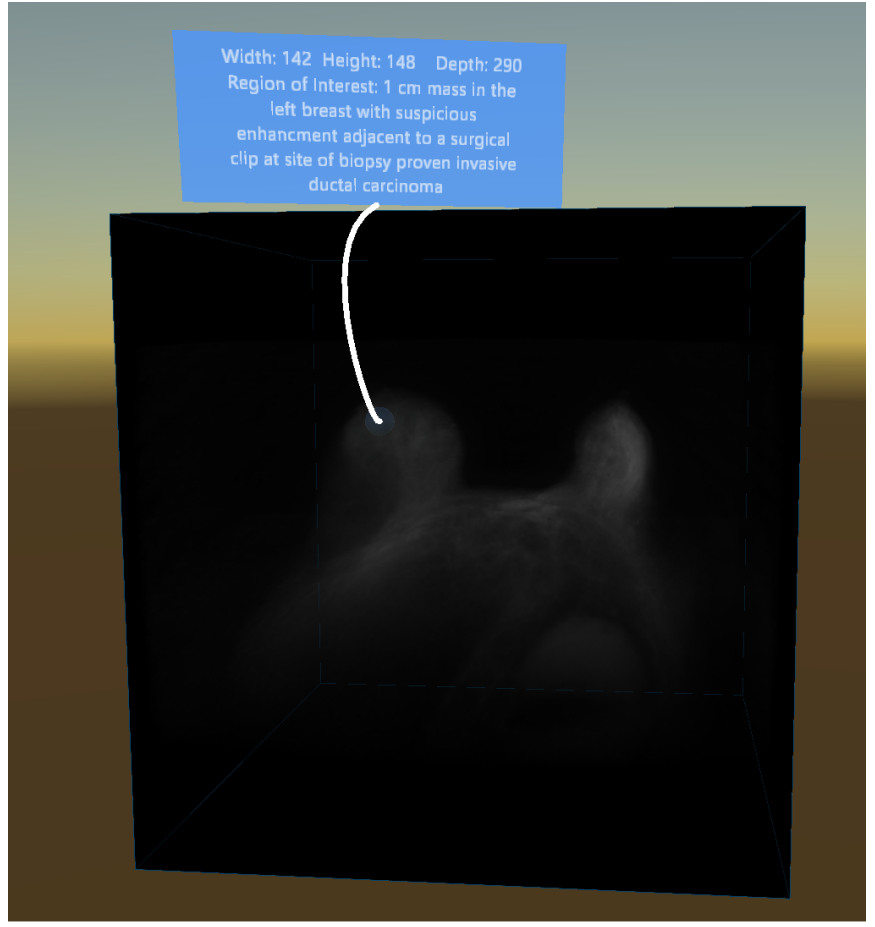

Fig. 1: Annotation of a point of interest (POI) with position and clinical notes

of volume exploration within XR freely controlled using hands gestures. Breast 3D will enable radiologists to be able navigate through the volume, slicing and segmenting regions from all angles to better understand breast composition.

\section{Diagnostic ToOls IN XR}

Our System, Breast3D, can be broken down into four core functionalities. These are 1) Voice commands; 2) Volume Interaction; 3) Volume Exploration; 4) Annotation. We will briefly explain these here.

\section{A. Voice Commands}

The most novel feature in comparison to other artefacts in literature is that Breast3D incorporates voice commands (a feature offered in Microsoft HoloLens but may not be supported by other manufacturers) to automate volume exploration. Currently our model offers the following voice command interactions with the reconstructed volume:

- Posterior - Orientates the volume to focus on the posterior region of the breast

- Anterior - Orientates the volume to focus on the anterior region of the breast

- Superior - Orientates the volume to focus on the superior region of the breast

- Inferior - Orientates the volume to focus on the inferior region of the breast

- Left - Orientates the volume to focus on the left region of the breast

- Right - Orientates the volume to focus on the right region of the breast 


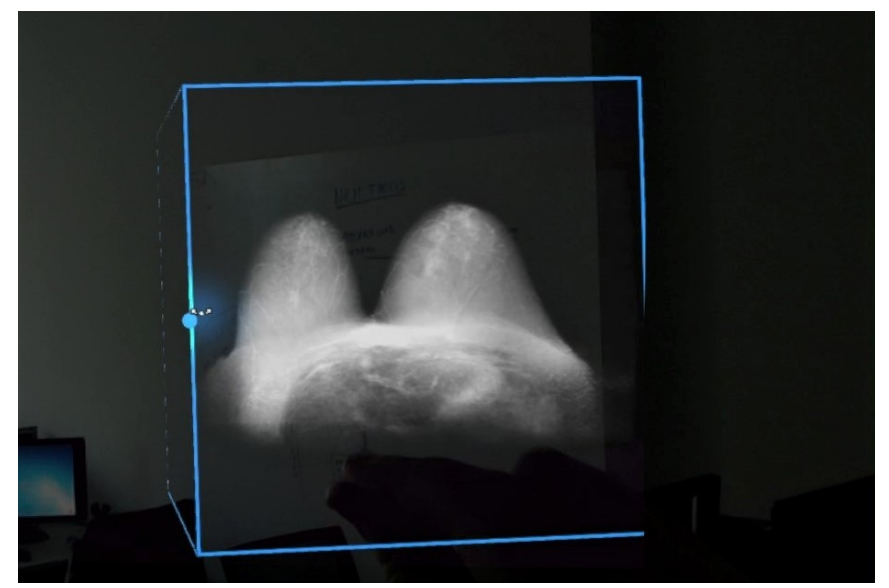

Fig. 2: User performing a pinch gesture of the hand to rotate a MRI breast volume in XR

- Annotate - Spawns a annotation within the volume

\section{B. Data Annotation}

Breast3D can accurately get the position of data points within the volume in $\mathrm{x}, \mathrm{y}$ and $\mathrm{z}$ axes to report an absolute point rather than relative description used in clinical reports. This point can be on the surface of the reconstructed model or deep embedded inside the stack accessed via our UI shown in figure 1. This provide the basis for further building a framework that is able to capture, save and share clinical observations for mammography.

\section{Volume Interaction}

Current systems used to examine radiological and oncological data implements various forms of user interfaces (UI) for $\mathrm{CT} / \mathrm{MRI}$ volume exploration. This is typically performed by the use of traditional methods relying on computer peripherals, i.e. a keyboard and mouse in conjunction with a 2D screens. $\mathrm{XR}$ does not just differentiate between a 3D to 2D visualisation, as it allows for previously unavailable ways of interaction by replacing the controller with hand gestures, replacing the need for a keyboard and mouse. Breast3D utilises a 3D UI which is fed and controlled by spatial motion data produced as a result of hand tracking. Research has shown that 3D UIs can enhance user understanding of clinical data [16], thus offering an more efficient interactive experience. Moreover, Breast3D can be expanded for use by multiple users at the same time, where various clinicians in the same and diverse locations can interact with the same volume at the same time. Hand gestures allows for key interactions within the volume. These includes the movement of the reconstructed volume in real space; scaling in size; rotation; colourization; zooming in and out as well as traversing through volume from all angles. The most significant feature of all is the ability to annotate a contradicted model in real space using a finger tip.

\section{Volume Exploration}

We have designed 3D UI shown in figure 3 for axis-aligned slicing, where the slicing plane is aligned to a specific axis.

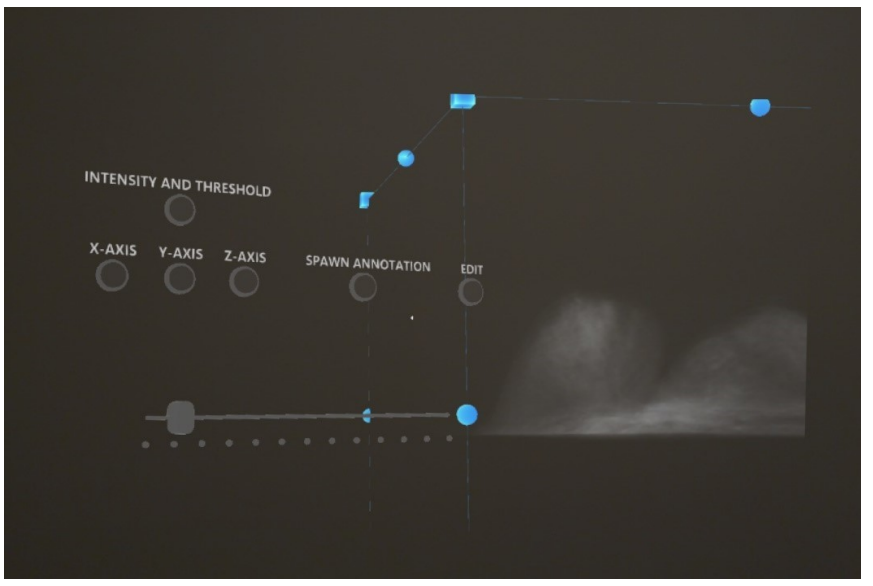

Fig. 3: 3D user interface in extended reality

We have used Microsoft mixed reality toolkit (MRTK) for our design, a publicly available library for building augmented reality applications. This allows for operations that would enable radiologists to explore the volume in a 3D real space; however, consistent with the UIs used in 2D clinical explorers. Users can control the reconstructed volume with hand gestures. The system uses bar sliders to enable search for unmasking (and potentially outlining) of ROI's, by traversing through the stack of the volume across $\mathrm{x}, \mathrm{y}$ and $\mathrm{z}$ axes. We demonstrate an example of exploration in figure 4, where the interaction elements discussed of Breast3D can be used together to isolate an ROI. First the breast MRI shown in figure $4 \mathrm{a}$ is rotated to the superior view shown in figure $4 \mathrm{~b}$, this can be done by the process shown in figure 2 by using pinch gesture or by utilising voice commands. Next utilising the 3D UI shown in figure 3 , the right breast can be isolated by axis aligned slicing by moving the sliders, the result of this is shown in figure $4 \mathrm{c}$. We further enhanced this by zooming in and pinching with both hands to move them apart.

\section{Discussion}

We have highlighted XR's specific use cases in medical image analysis of breast data, including its use for 3D visualisation, XR distributed radiology such as for multi-disciplinary team meetings, and specimen co-registration as shown by [4]. In addition, we have discussed the vast advantages of XR and how the technology improves upon the current practice in interactivity and visualisation. Furthermore the XR system extends to other radiological and oncological sub specialities. We discussed in detail the key functionalities XR systems provide and the advantages they offer over current practices within breast image analysis for mammography. We presented the potential improvements on current methods that can be made in visualisation, risk assessment and the unmasking of obscured masses when applying XR in breast radiomics.

Future research will apply existing image analysis techniques and develop new methods within XR, with the aim to specifically aid clinicians in diagnosis of breast cancer. This comprises of the further development of a interactive system to 

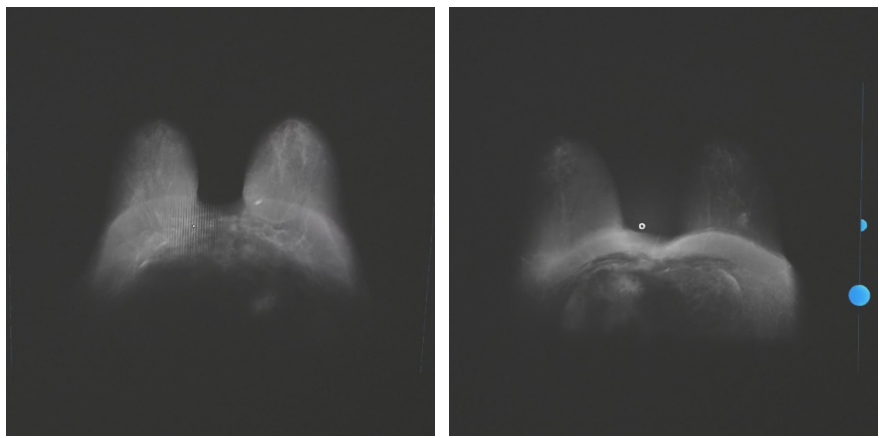

(a) Full view of breast MRI from (b) Full view of breast MRI from inferior position of the BI-RADS- the superior with adjustment to 6 scan

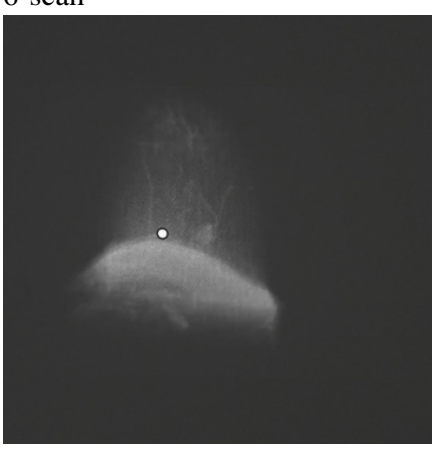

intensity values

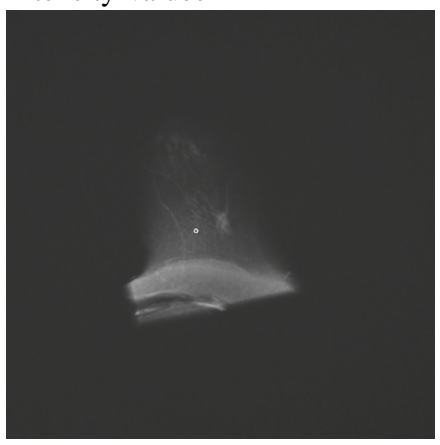

(c) View from the superior of the (d) View from the superior of the right breast after isolation by axis- right breast after isolation by axisaligned slicing

aligned slicing

Fig. 4: Isolation of a tumour of a BI-RADS 6 (biopsy proven mass) using Breast3D's interaction techniques

aid clinicians to overcome issues faced in mammography such as cancer masking, catching interval cancers earlier and finding developing focal asymmetries. Further development Breast3D will continue for its evaluation in a clinical setup. Adaptions can be made to convert Breast3D into a distributed system which is versatile for suitable for MDT meetings. Utilising our developed XR system we aim to quantify breast density with 3D volumetric data as opposed to current methods with $2 \mathrm{D}$ mammography. This will be done by differentiating between fat and non-fat component of the breast paranchyma and finding its percentage over the read 3D volume of the breast. The accumulation of this aims to overcome the weaknesses of using only 2D data to classify into BI-RADS when assessing breast cancer risk.

\section{REFERENCES}

[1] M. J. Yaffe et al., "Clinical outcomes of modelling mammography screening strategies," vol. 26, no. 12, p. 9, 2015.

[2] D. B. Douglas, J. M. Boone, E. Petricoin, L. Liotta, and E. Wilson, "Augmented reality imaging system: $3 \mathrm{~d}$ viewing of a breast cancer," vol. 2 , no. 9, 2016.

[3] J. Sutherland et al., "Applying modern virtual and augmented reality technologies to medical images and models," vol. 32, no. 1, pp. 38-53, 2019.

[4] M. G. Hanna, I. Ahmed, J. Nine, S. Prajapati, and L. Pantanowitz, "Augmented reality technology using Microsoft HoloLens in anatomic pathology," vol. 142, no. 5, pp. 638-644, 2018.
[5] D. B. Douglas et al., "Augmented reality and virtual reality: Initial successes in diagnostic radiology," 2018

[6] D. B. Douglas, J. M. Boone, E. Petricoin, L. Liotta, and E. Wilson, "Augmented reality imaging system: $3 \mathrm{~d}$ viewing of a breast cancer," vol. 2, no. 9, 2016.

[7] D. B. Douglas, C. A. Wilke, J. D. Gibson, J. M. Boone, and M. Wintermark, "Augmented reality: Advances in diagnostic imaging," vol. 1, no. 4, p. 29, 2017.

[8] P.-Y. Chiang, C.-C. Chen, and C.-H. Hsia, "A touchless interaction interface for observing medical imaging," vol. 58, pp. 363-373, 2019.

[9] L. Liberman and J. H. Menell, "Breast imaging reporting and data system (BI-RADS)," vol. 40, no. 3, pp. 409-430, 2002.

[10] B. C. Agency, "Discussion Guide: Breast Density ," 2019. Accessed: Sep. 2020.

[11] V. H. T. Limited, VolparaDensity.

[12] Hologic, Quantra, vol. 2.2.

[13] E. J. Aiello, D. S. Buist, E. White, and P. L. Porter, "Association between mammographic breast density and breast cancer tumor characteristics," vol. 14, no. 3, pp. 662-668, 2005.

[14] S. Y.-H. Chiu, S. Duffy, A. M.-F. Yen, L. Tabár, R. A. Smith, and H.H. Chen, "Effect of baseline breast density on breast cancer incidence, stage, mortality, and screening parameters: 25-year follow-up of a Swedish mammographic screening," vol. 19, no. 5, pp. 1219-1228, 2010.

[15] M. Sasaki et al., "Artificial intelligence for breast cancer detection in mammography: experience of use of the ScreenPoint Medical Transpara system in 310 Japanese women," pp. 1-10, 2020.

[16] J. C. Goble, K. Hinckley, R. Pausch, J. W. Snell, and N. F. Kassell, "Two-handed spatial interface tools for neurosurgical planning," vol. 28, no. 7, pp. 20-26, 1995 . 